\title{
UNA MIRADA A LAS INVESTIGACIONES CUALITATIVAS SOBRE JÓVENES, CONFLICTOS Y VIOLENCIA EN LAS ESCUELAS SECUNDARIAS DE AMÉRICA LATINA*
}

\section{A Look at Qualitative Research on Youth, Conflicts and Violence in LATIN American High Schools}

\section{Pablo Nahuel di Napoli ${ }^{* *}$}

Resumen: La violencia en las escuelas constituye una problemática social y educativa que es noticia en los medios de comunicación, núcleo de políticas públicas y, también, objeto de estudio dentro del campo académico. En el siguiente artículo nos proponemos realizar una revisión bibliográfica de las investigaciones sobre violencias en las escuelas llevadas a cabo dentro del campo socioeducativo desde una perspectiva metodológica cualitativa. Para ello, analizamos en clave comparativa hallazgos comunes, diferencias y particularidades relevantes de los estudios a partir de cuatro núcleos temáticos: a) la violencia como juego, b) la violencia en el marco de los conflictos, c) el contexto social de

\footnotetext{
Una versión preliminar de este trabajo fue presentada en las IV Jornadas Nacionales y II Latinoamericanas de Investigadores/as en Formación en Educación. Este artículo es el resultado del revelamiento de investigaciones realizado en el marco de una beca doctoral, periodo 20112016, y otra postdoctoral, periodo 2016-2018, otorgadas y financiadas por el Consejo Nacional de Investigaciones Científicas y Técnicas de Argentina.

${ }^{* *}$ Doctor en Ciencias Sociales por la Universidad de Buenos Aires, UBA, Argentina. Licenciado y profesor en Sociología por UBA. Postdoctorado en el Departamento de Investigaciones Educativas del Cinvestav IPN, México. Docente de la Facultad de Filosofía y Letras y de la Facultad de Ciencias Sociales de UBA. Becario postdoctoral del Consejo Nacional de Investigaciones Científicas y Técnicas, Conicet, con sede en el Instituto de Investigaciones en Ciencias de la Educación de UBA. Temas de investigación: convivencia, conflictos y violencias en las escuelas; jóvenes, subjetividad y escuela secundaria; sociabilidades escolares y procesos de estigmatización entre jóvenes. Correo e.: pablodinapoli@filo.uba.ar Fecha de recepción: 0410 17; 2a. versión: 2905 18; Fecha de aceptación: 310518.
}

(cc) BY-NC-ND Páginas 9-38. 
la violencia, y d) la violencia como modo de relacionarse con el otro. Nuestro propósito es dar cuenta de los ejes de indagación abordados en nuestra región con el fin de contribuir al diseño de programas de convivencia, así como también a la exploración de nuevas áreas de vacancia.

Palabras clave: escuela, estudiantes, conflictividad, Latinoamérica.

Abstract: As a social and educational problem, school violence becomes present as news in the media, is core of public policies and also an object of study within the academic field. In the following article we review research in the socioeducational field addressing school violence from a qualitative methodological perspective. We analyze, in comparative key, common findings, differences and relevant particularities of these studies considering four thematic groups: a) violence as a game, b) violence in the context of conflict, c) the social context of violence and, d) violence as a way of relating to others. This article accounts for the subjects of inquiry addressed in our region in order to contribute to the design of mediation programs, as well as to explore new areas of vacancy.

Keywords: school, student, conflict, Latin America.

\section{Introducción}

En la actualidad, la violencia en el ámbito escolar constituye una problemática social y educativa que es noticia en los medios de comunicación, núcleo de diversas políticas públicas y, también, objeto de estudio dentro del campo académico. Como es sabido, esta temática no emerge espontáneamente en la agenda pública, sino que es el resultado de un trabajo político de construcción y selección de un determinado aspecto de la realidad como problema social. Como sostiene Martín Criado (2005: 87), “esta construcción no la realiza la sociedad: siempre tiene, como actores privilegiados, determinados 
grupos sociales u organizaciones que se esfuerzan por imponer la percepción de una determinada situación como problema social”.

Los medios de comunicación constituyen uno de los actores primordiales en los procesos de construcción de sentidos y sensibilidades en nuestras sociedades. Como afirma Bourdieu (1997), éstos detentan un gran poder simbólico gracias a su monopolio sobre los instrumentos de producción y de difusión en gran escala de la información. En este marco, diversos autores (Spósito, 2001; Furlán, 2005; Brener, 2009) señalan que la consolidación de la violencia en las escuelas como objeto de estudio estuvo atravesada por la alta visibilidad que le dieron los medios de comunicación en las últimas dos décadas. La escuela aparece representada, en muchos casos, como un espacio público acechado por la violencia provocada por jóvenes. A partir de una revisión de investigaciones realizadas en América Latina sobre el tratamiento mediático de hechos de violencia en las escuelas, Saez (2015: 147) observó cómo los discursos mediáticos “... presentan una figura estigmatizada de juventud, negando la complejidad de la condición juvenil e invisibilizando el ejercicio de la ciudadanía por parte de estos actores".

En la órbita de los organismos gubernamentales y multilaterales en varios países de Latinoamérica se han diseñado diversas políticas y programas orientados a reducir la violencia en el ámbito escolar. El programa Escuela Segura ${ }^{1}$, en México; el convenio Construyendo una Comunidad Educativa más Segura, en Uruguay² la creación del Batallón escolar, en el Distrito Federal de Brasil, son ejemplos de políticas pensadas desde el paradigma de la seguridad. En el caso de Argentina

\footnotetext{
${ }^{1}$ Desde 2007 el Programa Escuela Segura, tiene como “... objetivo que las escuelas públicas de educación básica del País se constituyan en espacios libres de violencia, adicciones y delincuencia, fortaleciendo el desarrollo integral de los alumnos en la promoción de actitudes y valores en la cultura de la prevención y en la seguridad escolar..." (Bases de operación del programa nacional escuela segura, 2008, p. 2). Actualmente en México también están en marcha el Programa Nacional de Convivencia Escolar, focalizado en situaciones de hostigamiento; Construye-t, centrado en la Educación Media Superior, o los comités de desaliento a las prácticas que generen violencia entre pares en el marco de los Consejos Escolares de Participación Social (Romo, Nashiki, Cervantes y Rivera, 2016).

${ }^{2}$ El año 2006 se firmó el convenio Construyendo una Comunidad Educativa más Segura entre el Consejo de Educación Secundaria, CES, y el Ministerio del Interior. Dicho “... convenio se sustentaba en la necesidad del CES de contar con personal policial y en el requerimiento del Ministerio de aumentar el nivel educativo del personal subalterno" (Viscardi y Alonso, 2013: 63).
} 
destacamos la creación en 2004 del Observatorio Argentino de Violencia en las Escuelas y el Programa Nacional de Convivencia Escolar ${ }^{3}$ con una perspectiva alternativa.

Dentro del campo académico, en el plano internacional podemos encontrar estudios pioneros desde mediados de la década de 1970 en países como Francia, Estados Unidos, Noruega. Sin embargo, es recién en las vísperas del nuevo siglo cuando comienza a consolidarse un abordaje más sistemático de la problemática y a desarrollarse nuevas investigaciones en países como España, Brasil, México y Uruguay (Kaplan y García, 2006). En el caso de Argentina, a excepción de trabajos pioneros (Kornblit, Mendes Diz y Frankel, 1991), es hace poco más de una década que empezaron a llevarse a cabo estudios sistemáticos sobre el tema (Duschatzky y Corea, 2002; Kaplan, 2006, 2013; Bleichmar, 2008; Kornblit, 2008; Míguez, 2008; OAVE, 2008; Paulín y Tomasini, 2008, 2014; Noel, 2009; D’Angelo y Fernández, 2011; Míguez, Gallo y Tomasini, 2015).

En un trabajo anterior (di Napoli, 2016a) hemos realizado una revisión analítica del campo de las investigaciones sobre violencias en el ámbito escolar destacando tres grandes perspectivas a nivel mundial, las cuales diferenciamos por su modo de abordaje y la delimitación que hacen de la temática. Desde un enfoque que denominamos criminológico, se restringe la violencia en las escuelas a cuestiones ilegales, punitivas y de seguridad tipificadas por el código penal y se la relaciona con comportamientos de tipo "protodelictivos". Desde la perspectiva psicoeducativa, que focaliza su estudio en el bullying, se concibe la violencia en términos de hostigamiento a través de actos intencionados reiterados en el tiempo que causan dańo o hieren a determinados individuos. Este enfoque centra sus indagaciones sobre las características psíquicas y actitudinales del agresor y la víctima reduciendo las situaciones de maltrato escolar a una lógica dicotómica de víctima y victimario en el marco de relaciones asimétricas de poder. Por último, el enfoque socioeducativo contempla como dimensiones centrales los contextos

\footnotetext{
${ }^{3}$ El primero tiene como propósito sumar esfuerzos para el estudio de la temática de la violencia en las escuelas, contribuir a la consolidación de las prácticas democráticas en el ámbito educativo y la construcción de espacios de ciudadanía; el segundo busca ofrecer recursos en el ámbito de las normativas escolares, la supervisión del sistema educativo y el vínculo entre la escuela y la familia.
} 
socioculturales e institucionales donde se producen las violencias y los sentidos que los actores les otorgan. En estas investigaciones se concibe una definición ampliada de la violencia que incluye un conjunto heterogéneo de actos que, sin ser necesariamente ilegales, son vividos como violentos por quienes los padecen.

\section{Método}

En el presente artículo nos proponemos realizar una revisión de la literatura, la cual no pretende ser un análisis bibliométrico, sobre las investigaciones ancladas en el último enfoque mencionado: el "socioeducativo". Como en toda revisión bibliográfica, aquí realizamos una selección interesada de los estudios. Los criterios de selección utilizados fueron los siguientes: a) investigaciones empíricas cualitativas, b) llevadas a cabo en países latinoamericanos, c) en escuelas secundarias — de educación media—, y d) que indaguen sobre los sentidos y prácticas que los estudiantes construyen en torno a la violencia en las escuelas.

La búsqueda bibliográfica consistió en la consulta de tres catálogos en línea de revistas científicas de amplia relevancia en la región: SciELO, Redalyc y SCOPUS. La consulta se realizó a partir de la siguiente estructura de TAG's: violencia AND escuela OR bachillerato AND estudiantes OR alumnos AND NOT bullying. En total se registraron 388 documentos: 183 en SciELO, 131 en SCOPUS y 74 en Redalyc. Luego de excluir los artículos repetidos y seleccionar las investigaciones que cumplían con los criterios establecidos más arriba el corpus quedo conformado por 25 artículos/investigaciones.

Figura 1. Selección de catálogos en línea de revistas científicas y articulos de estudio.

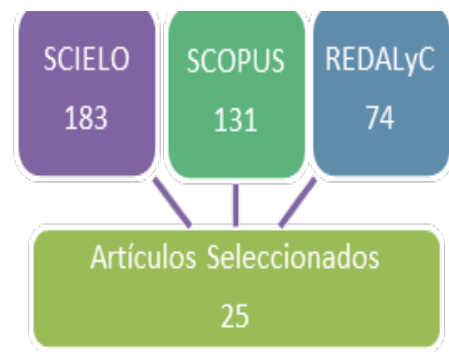

Fuente: Elaboración del autor 
Cuando se encontró más de un artículo por autor que reflejaba resultados de una misma investigación se procedió a seleccionar aquel más pertinente para los objetivos del presente trabajo. En el caso de los artículos que eran producto de tesis de postgrado se trató de acceder a las mismas para ampliar el contenido de los hallazgos producto de la investigación.

Tabla 1. Lugar y año de articulos seleccionados.

\begin{tabular}{|l|c|c|c|c|c|c|c|c|c|c|c|c|c|c|}
\hline \multirow{2}{*}{$\begin{array}{l}\text { País donde } \\
\begin{array}{l}\text { se realizó } \\
\text { el estudio }\end{array}\end{array}$} & \multicolumn{1}{|c|}{2001} & 2002 & 2003 & 2005 & 2007 & 2009 & 2011 & 2012 & 2013 & 2014 & 2015 & 2016 & 2017 & Total \\
\hline Brasil & 1 & & 1 & 1 & 1 & & & & & 1 & & 2 & 1 & 8 \\
\hline Argentina & & & & 1 & & 1 & 1 & & 3 & & 1 & & & 7 \\
\hline México & & & & 1 & & & & 2 & & 1 & & & 1 & 5 \\
\hline Chile & & & & 1 & & & 1 & & & & & & & 2 \\
\hline Uruguay & & 1 & & & & & & & & & & & & 1 \\
\hline Bolivia & & & & & & & & & & 1 & & & & 1 \\
\hline Colombia & & & & & & & 1 & & & & & & & 1 \\
\hline \multicolumn{1}{c}{ Total } & 1 & 1 & 1 & 4 & 1 & 1 & 3 & 2 & 3 & 3 & 1 & 2 & 2 & 25 \\
\hline
\end{tabular}

Fuente: Elaboración del autor

En la tabla anterior podemos observar que la mayor cantidad de investigaciones se realizaron en Brasil (8), seguido por Argentina (7) y México (5). La Revista Mexicana de Investigación Educativa ha sido la que más artículos (4) ha editado sobre la temática desde la perspectiva analizada en este trabajo. Cabe destacar que dicha revista ha dedicado en el año 2005 dos números temáticos, núms. 26 y 27, denominados "Problemas de indisciplina y violencia en la escuela", en los cuales agrupó investigaciones realizadas en Argentina, Brasil y México.

Los instrumentos de recolección de datos privilegiados fueron las entrevistas individuales en profundidad y/o los grupos focales. Algunos estudios también se nutrieron de observaciones sistemáticas de clases y/o de diversos espacios escolares (Di Leo, 2009; Mejía Hernández, 2012; Tomasini, Domínguez y Peralta, 2013; Paulín, 2015). Otras estrategias 
utilizadas fueron la etnografía (Maldonado, 2005; Chuquilin Cubas y Zagaceta Sarmiento, 2017), la narrativa (Duschatzky, 2013; Conde Flores, 2014), historias de vida (Velázquez Reyes, 2005), y el análisis de redes semánticas (García-Villanueva, De la Rosa-Acosta y CastilloValdés, 2012).

\section{Las investigaciones en América Latina}

Consideramos pertinente abordar las situaciones de violencia que involucran a estudiantes o grupos de estudiantes en el marco de una matriz societal donde se entrecruzan los condicionamientos sociales e institucionales y las experiencias y percepciones subjetivas (Kaplan, 2009). Creemos que no basta con revelar los "hechos violentos", conocer sus características e identificar a los protagonistas, sino que es también necesario comprender la dinámica y significación de la violencia como un fenómeno relacional que emerge en configuraciones sociales específicas.

Bajo esta premisa, nos preguntamos por los sentidos y las experiencias de subjetivación que construyen los jóvenes escolarizados en torno a la violencia en sus escuelas. En nuestro recorrido por las investigaciones realizadas en diversas latitudes de América Latina nos interesa conocer cuáles son las percepciones de los estudiantes de educación media sobre la violencia y qué sentidos le otorgan con el objetivo de contribuir a la resolución pacífica de conflictos y la elaboración de estrategias de convivencia escolar. Para lo anterior, nos proponemos analizar en clave comparativa hallazgos comunes, diferencias y particularidades relevantes que arrojan los estudios realizados en la región a partir de cuatro ejes: a) la violencia como juego, b) la violencia en el marco de los conflictos, c) el contexto social de la violencia y, c) la violencia como modo de relacionarse con el otro.

\section{La violencia como juego}

Varias investigaciones hacen alusión a la naturalización o banalización de la violencia no tanto debido a una valorización o aprobación de su uso, sino más bien a un modo de sociabilidad lúdica. Muchas de las prácticas o formas de interacción entre los estudiantes que los adultos perciben como violentas o que podrían ser incluidas dentro de alguna 
definición analítica del término, los jóvenes las describen como parte de un contexto lúdico, como una forma de estar juntos.

En México, encontramos dos investigaciones que trabajaron con jóvenes tipificados como "violentos" (Chuquilin Cubas y Zagaceta Sarmiento, 2017) o "problemáticos” (Mejía Hernández, 2012) por los agentes educativos, donde observaron prácticas intersubjetivas lúdicas bruscas regladas por el principio que Saucedo (2006) denominó como "llevarse y aguantarse". Mejía Hernández, desde el enfoque de la psicología cultural, indagó sobre las formas de relacionarse de los estudiantes de cuatro planteles públicos de la Ciudad de México durante un ciclo escolar a partir de observaciones, entrevistas y grupos focales a docentes y estudiantes. Los grupos de estudiantes calificados como "pesados" o problemáticos" percibían como común relacionarse a través de insultos y rivalidades con los que "se llevan pesado", siempre y cuando la interacción sea simétrica. "La naturalización de tales comportamientos como 'juegos', aun cuando saben que son objeto de desaprobación y sanción escolar, ... reduce la posibilidad de que estos alumnos los visualicen como incivilidades" (Mejía Hernández, 2012: 64-65).

Por su parte, Chuquilin Cubas y Zagaceta Sarmiento (2017), en un estudio reciente de corte etnográfico, también en una escuela secundaria pública de la Ciudad de México, observaron, además de diferentes tipos de interacción consideradas por los estudiantes parte del "relajo" - como formar ruedos y hacer bola en el patio o armar alborotos y empujarse-, un juego con tradición denominado "Pamba". Se trata de:

... una actividad lúdica que une a grupos de amistades de barrio, compañeros y compañeras de aula, hermanos hermanas y familiares. En el caso de esta escuela, la pamba es una práctica común y su intencionalidad difiere en los grupos que lo practican. Algunos lo hacen para divertirse y hacer relajo cuando el personal docente no asiste a clase. Otros, para poner a prueba su resistencia al dolor, ya que las reglas del juego implican propinar y recibir golpes rudos (Chuquilin Cubas y Zagaceta Sarmiento, 2017: 7). 
En Argentina, Paulín (2015) y Tomasini, Domínguez y Peralta (2013), quienes realizaron investigaciones en escuelas secundarias de gestión estatal y gestión privada de la ciudad de Córdoba, encontraron lógicas de interacción que contenían diferentes tipos de agresión - física, verbal, simbólica-, pero que para los estudiantes constituían formas de “joder" y "jugar a molestarse". Paulín (2015) sostiene que esta forma de sociabilidad tiene un papel decisivo en la constitución de identidad, la regulación de las emociones y los modos de construcción de aceptación o rechazo de ciertas diferencias sociales y culturales entre las personas jóvenes. En este sentido, Tomasini, Dominguez y Peralta (2013) concluyen que estos comportamientos son parte de la sociabilidad cotidiana de los jóvenes en la escuela a partir de la cual se (re) conocen, instituyen códigos de convivencia y, también, valoraciones sobre sí mismos y sobre los otros.

De modo similar, en una investigación que realizamos en dos escuelas secundarias de gestión estatal de la Provincia de Buenos Aires (di Napoli, 2016b), nos encontramos con dos grupos de estudiantes varones, uno en cada escuela, que edificaron un modo de sociabilidad a través de prácticas ritualizadas que caracterizamos analíticamente como violentas, sin ser reconocidas por ellos de esa forma. En el grupo de estudiantes, que provenía de sectores populares, las interacciones lúdicas a las que denominaban "malteadas", requerían del uso de la fuerza física, en cambio el otro grupo de alumnos oriundos de sectores sociales medios tenían pautado como juego "hacerse pasar calor", el cual consistía en realizar diferentes prácticas de humillación. Dado el grado de confianza grupal adquirido, en ninguno de los dos grupos interpretaban que quienes usaran la fuerza física o se burlaran, lo hicieran con la intención de lastimar. Tampoco consideraban que quien recibiera los golpes o fuera objeto de burlas podría sentirse herido.

En nuestra investigación pudimos concluir que las percepciones de los estudiantes en torno a la violencia en el espacio escolar son relacionales y contextuales. Es decir, que están en mayor medida permeadas por la calidad de los vínculos que construyen con sus pares y por los escenarios en los cuales se desarrollan, que por tipo de práctica en sí misma o la concepción a priori que tengan sobre la violencia. 
En esta misma línea interpretativa se enmarcan Coarite y Carrillo (2014), quienes realizaron un estudio a través de grupos focales con estudiantes de dos unidades educativas de la ciudad de El Alto, en La Paz, Bolivia. Los investigadores afirman que el devenir de los conflictos entre los estudiantes depende del contexto mismo de la interacción en el cual interpretan si existió, o no, la intención de agredir o desafiar. Se evalúa el

cómo - por ejemplo, hablando con gritos, de modo despectivo y desafiante, usando diminutivos o haciendo bromas amistosas-, quién - por ejemplo, si fue un varón, una mujer, su mejor amigo/a o un/a compañero con quien se tuvo conflictos previamente- y dónde - por ejemplo, mientras participan de un grupo en clases o de un juego fuera del aula - se hayan utilizado los ademanes y dicho las palabras (Coarite y Carrillo, 2014: 184).

De este modo, consideramos relevante identificar los comportamientos, contextos y circunstancias por las cuales una situación deja de ser un juego para pasar a ser percibida como ofensiva. Distintas investigaciones han marcado la alta importancia que los estudiantes le atribuyen a su juicio sobre la "intención” de la acción del otro (Potocnjak, Berger y Tomicic, 2011; Mejía Hernández, 2012; Tomasini et al., 2013; Coarite y Carrillo, 2014; di Napoli, 2016b).

\section{La violencia en el marco de los conflictos}

Abramovay, a partir de dos investigaciones realizadas en escuelas de gestión estatal y gestión privada de diferentes ciudades del Brasil (Abramovay, 2005; Abramovay, Cunha y Calaf, 2009), sostiene que los motivos por los cuales los estudiantes se agreden físicamente pueden ser variados y parecer absurdos o fútiles para aquellos que no están inmersos en las lógicas juveniles. Incluso para quienes las sufren tampoco está siempre claro el motivo:

El ambiente entre los estudiantes es a menudo impregnado de relaciones conflictivas de todo tipo, incluyendo agresiones verbales, físicas, 
humillaciones y falta de respeto. Son modos de relación, muchas veces tomadas como típicas de la adolescencia/juventud, que no pueden ser visto a priori como incivilizadas, pero que pueden llegar a socavar las buenas relaciones entre los alumnos, tornando el ambiente más propicio a conflictos y resoluciones violentas (Abramovay et al., 2009, pp. 103-104).

Estas acciones pueden ser parte de un juego o sonar como burlas. Sin embargo, no siempre es comprendido de la misma forma por todos los actores. Insultarse puede ser una forma común de tratarse entre los estudiantes pero, también, puede expresar enojo frente a alguna situación, un modo de buscar pelea a través de la ofensa o una forma de divertirse al humillar al otro.

Las distintas investigaciones reseñadas han dado cuenta de diferentes tipos de conflicto o situaciones conflictivas que podían ser percibidas como violentas: pertenecer a un determinado barrio (Araújo, 2001; di Napoli, 2013); rivalidades deportivas, particularmente futbolísticas; (Abramovay et al., 2009; di Napoli, 2016b); prácticas de discriminación o humillación (Di Leo, 2009; di Napoli, 2013; Tomasini et al., 2013; Salles, Silva, Castro y Fernandez Villanueva, 2014; Schilling y Angelucci, 2016; Giordani et al., 2017), entre otras, pueden ser eje de conflicto y constituirse en formas de violencia.

Entre los diversos motivos de conflictos que señalan las investigaciones destacamos particularmente tres que han sido mencionados en casi todas. El primero refiere a lo que los estudiantes denominan "mirar mal", "mirar feo" o "mirada de barrida". Di Leo (2009), en su investigación en dos escuelas secundarias de la Ciudad Autónoma de Buenos Aires, sostiene que en el "mirar mal" se manifiesta una "lucha" en torno a la aprobación del par — pareja, amigo/a, compañero/a—. Esta necesidad de reconocimiento es posible vincularla “... con la construcción y mantenimiento de la 'confianza en las relaciones cara a cara', en tanto pilar fundamental de los procesos de subjetivación y de constitución de la 'seguridad ontológica' de las personas jóvenes en las diversas esferas de la vida cotidiana” (ídem: 63). La violencia contra el otro expresa: 
... un primer momento de la lucha por el reconocimiento a partir de un doble movimiento: a) la negación de la corporeidad del otro, vista como obstáculo para el reconocimiento del sí mismo como sujeto, y simultáneamente, b) la afirmación de la propia subjetividad, al demostrar en la lucha — que puede poner en riesgo su propia corporeidad - que está más allá de la mera objetualidad en que lo pone la mirada degradante del otro (ídem: 63).

El "mirar mal" atraviesa la sociabilidad de los estudiantes en el espacio escolar y trae aparejado una serie de conflictos. A través de la mirada se ponen en juego diferentes aristas de la individualidad: el reconocimiento como persona, la pertenencia a un determinado grupo y la construcción subjetiva de una identidad propia.

El segundo motivo refiere al insulto a algún familiar, pero particularmente dirigido hacia las madres. Por ejemplo, la mención a la madre entre las interacciones lúdicas de los estudiantes provoca que el equilibrio de un juego se rompa para pasar a una situación de violencia producto de la agresión verbal recibida. Mejía Hernández (2012: 64) afirma que la figura materna es sagrada: "fallarle al respeto mediante una 'mentada de madre' es un antivalor que se interpreta como ataque que amerita desagravio".

El tercer motivo de conflicto entre estudiantes se relaciona con los enamoramientos, noviazgos, celos, infidelidades o juegos de seducción. En plena adolescencia la sexualidad moviliza toda una serie de emociones, aprendizajes, códigos de relacionamiento y conflictos. La investigadora argentina Maldonado (2005), quien realizó una investigación etnográfica en una escuela de la ciudad de Córdoba, problematiza esta cuestión al analizar la rivalidad entre un grupo de estudiantes mujeres por un chico. Se trataba de una estudiante nueva que al conformar una pareja con uno de los compañeros del curso quebró el equilibrio que existía al interior de un grupo de amigas en el que a dos de ellas les gustaba el mismo compañero.

Por otra parte, varias investigaciones han marcado diferencias entre géneros respecto de los motivos de pelea, las formas de conflictividad y los tipos de violencia. Algunas de ellas focalizaron sus indagaciones sobre 
las diferencias entre estudiantes mujeres y estudiantes varones (Mejía Hernández, 2013), incluyeron una perspectiva de género (Tomasini et al., 2013) o abordando las interacciones entre un género específico (Torres-Castro, 2011).

Sin embargo, no encontramos unanimidad en las diferenciaciones que se establecen por género. Por ejemplo, en el caso de las investigaciones realizadas en Brasil (Abramovay, 2005; Ribolla \& Fiamenghi Jr., 2007; Salles et al., 2014; Happ Botler, 2016; Giordani et al., 2017) y Uruguay (Viscardi, 2002) se destaca como uno de los principales motivos de pelea entre las mujeres las disputas por un varón; en cambio en Argentina (Tomasini et al., 2013; Paulín, 2015; di Napoli, 2016b) y México (Mejía Hernández, 2013), los investigadores sostienen que a pesar de que entre los estudiantes existe un discurso hegemónico respecto a que ese tipo de conflictos es propio del género femenino, se producen tanto entre mujeres como entre varones al enfrentarse por la causa de una persona del sexo opuesto.

Otra cuestión por señalar es el uso de la violencia física tradicionalmente asociada a la construcción de masculinidad. Las investigaciones realizadas en Brasil muestran que las agresiones físicas son protagonizadas casi con la misma frecuencia por varones que por mujeres (Abramovay, 2005; Ribolla \& Fiamenghi Jr., 2007; Salles et al., 2014; Happ Botler, 2016; Giordani et al., 2017). Mientras que en Chile (Potocnjak et al., 2011), México (Velázquez Reyes, 2005; García-Villanueva et al., 2012) y Uruguay (Viscardi, 2002), sin negar la existencia de enfrentamientos con uso de la fuerza física entre mujeres, asocian al género femenino con violencias de tipo más relacional expresadas en formas de exclusión o agresiones verbales. En el caso de los varones identifican como más frecuentes los juegos y las peleas con uso de la fuerza física. Sin embargo, investigaciones recientes desarrolladas en Argentina (di Napoli, 2016b; Paulín, 2015; Tomasini et al., 2013) y México (Mejía Hernández, 2013; Chuquilin Cubas y Zagaceta Sarmiento, 2017), dan cuenta de la existencia de numerosas situaciones de violencia física entre mujeres. Asimismo, las investigaciones realizadas en Argentina señalan que el uso de la fuerza física para dirimir conflictos por parte de estudiantes mujeres está más presente en aquellas escuelas que reciben 
alumnos de sectores sociales populares. Por último, cabe destacar el consenso relacionado con que la violencia física entre mujeres, aunque pueda ser menos frecuente, genera mayor impacto especular entre los distintos actores de la comunidad educativa.

\section{El contexto social de la violencia}

A raíz del uso diferencial de la violencia física por parte de las estudiantes mujeres según el origen socioeconómico que observan algunas pesquisas realizadas en Argentina, resulta pertinente reflexionar sobre la relación —no causal— que establecen los estudios entre la violencia que acontece en las escuelas y el contexto social en el cual se encuentran ubicadas. Varias de las investigaciones reveladas llevaron a cabo el trabajo de campo en escuelas a la que asistían estudiantes provenientes de sectores sociales vulnerabilizados y que estaban ubicadas en áreas urbanas con altos índices de violencia (Torres-Castro, 2011; Duschatzky, 2013; Salles et al., 2014). Asimismo, ciertas investigaciones, particularmente en Brasil (Araújo, 2001) y México (Conde Flores, 2014) se focalizaron también en escuelas ubicadas en ciudades con altos índices de violencia vinculada al crimen organizado.

Duschatzky (2013: 353), quien realizó investigaciones en escuelas urbano-marginales de la provincia de Buenos Aires, afirma que “... en condiciones sociales de exclusión y de banalización de la muerte, la violencia remite entre otras cosas a un estilo de estar juntos, a una forma de vivir y desafiar el tiempo". Así, la violencia puede constituirse en un modo de vivir, de estar con el otro y de construir subjetividad.

A una conclusión similar llega Conde Flores (2014), quien llevó a cabo un estudio en diez escuelas de cinco ciudades con altos índices de criminalidad y violencia vinculada al crimen organizado en México. La investigadora sostiene que:

Cuando se vive en entornos violentos, sin esperanza de que la situación cambie, parece que el aprendizaje de la violencia ayuda a sobrevivir, a llevarla en paz con la banda del barrio, a ganarse el respeto. Aquí se cruza la construcción de las identidades adolescentes y de los sentidos de pertenencia con los modelos imperantes, pero también con los 
disfraces, con la pose que se adopta por instinto de conservación hasta que quedan atrapados en su camuflaje (Conde Flores, 2014: 5).

Justamente, la pesquisa realizada por Carla Araújo (2001) indaga sobre las marcas de la violencia que operan en la construcción de identidad de estudiantes que viven en un barrio socialmente vulnerable y conviven en la escuela con jóvenes de otros barrios con mejor poder adquisitivo. Se trata de un barrio problemático de Belo Horizonte, Brasil, con altos índices de delitos y homicidios donde, también, existen conflictos territoriales vinculados con la venta de droga. La violencia que acontece en el barrio es una de las marcas que se relaciona con la identidad de los estudiantes que residen allí. A su vez, la inseguridad e incertidumbre que los jóvenes viven en su barrio hace que el miedo sea uno de los sentimientos que atraviese su subjetividad.

Pertenecer a ese barrio tiene consecuencias contrapuestas en la relación con sus compañeros. Ser oriundos de una favela les propicia respeto y fama de "tener calle", ser valientes y a su vez ser temidos; pero, también, los hace objeto de estigmatización por parte de sus pares.

Araújo (2001) concluye que el contexto de violencia que padecen los estudiantes en su barrio hace que aumente su naturalización. Esto se ve reflejado en que sólo consideran una situación como violenta cuando hay armas o muertos de por medio. El temor que generan en sus compañeros por su procedencia hace que tengan que realizar un esfuerzo extra por ganarse su confianza. A su vez, estos jóvenes no logran construir una identidad a partir de su barrio, sino que se sienten avergonzados de vivir allí.

En este marco, algunos estudios sostienen que la violencia en el ámbito escolar puede ser interpretada como consecuencia de una violencia social "extramuros" que ingresa en la escuela por la pobreza, la marginación social, la delincuencia. y que se agrava en América Latina por la expansión del tráfico de drogas y el crimen organizado (Conde Flores, 2014; Salles et al., 2014; Giordani et al., 2017). La investigadora colombiana Torres Castro (2011: 392) afirma que: 
... el alto impacto social de una vida comunitaria conflictiva y un espacio barrial propicio para la conformación de pandillas, en la vida de las adolescentes, pues las afecta de manera sistemática y las hace vulnerables a la solución violenta de los conflictos, lo que provoca que la violencia escolar femenina se manifieste de acuerdo con lo aprendido en estos grupos.

Sin embargo, otras investigaciones (Kaplan, 2011; Chuquilin Cubas y Zagaceta Sarmiento, 2017), incluso aquellas realizadas en zonas socialmente vulnerables (Araújo, 2001; Duschatzky, 2013), matizan la relación entre el contexto social y las violencias que acontecen en el ámbito escolar aduciendo cuestiones vinculadas a las lógicas institucionales de las escuelas y las trayectorias y biografías de los actores de la comunidad educativa — estudiantes, padres y agentes escolares.

Resulta necesario aclarar y evitar asociar mecánicamente los contextos de vulnerabilidad social de las escuelas con el acaecimiento de situaciones de violencia en su seno. Míguez (2008) sostiene que, en el caso argentino, las investigaciones cuantitativas no revelan diferencias significativas entre las condiciones sociales y la violencia que acontece en las escuelas, mientras no se trate de formas extremas como muertes o lesiones con armas. ${ }^{4}$ Sin embargo, los estudios de corte cualitativo, que generalmente se han centrado en escuelas rodeadas de alta conflictividad social donde la población vive en condiciones gravosas, sí encuentran recurrentemente vinculaciones entre pobreza y violencia en las escuelas. De este modo, el autor sostiene que "mientras la mirada estadística nos indica la proporción del problema, la etnografía nos señala las excepcionalidades a las que deberíamos prestar atención” (Míguez, 2008: 25).

Por otra parte, dos investigaciones realizadas en Brasil que analizaron la relación entre conflicto, violencia e injusticia en las escuelas, pusieron el foco en la contraposición entre la cultura escolar y la

\footnotetext{
${ }^{4}$ A diferencia de otros países como Estados Unidos, Francia y Noruega, en Latinoamérica los estudios sistemáticos a nivel nacional sobre el tema son casi nulos. A inicios del presente siglo empezaron a realizarse revelamientos en Argentina, Brasil, Chile, Colombia y México, de los cuales solamente en Argentina y Chile fueron replicados al menos tres veces. En el caso de Argentina actualmente dichos revelamientos han sido discontinuos.
} 
cultura juvenil, el conflicto cultural de tipo generacional entre jóvenes estudiantes y docentes adultos o lo que Charlot (2002) calificó como violencia hacia la escuela en respuesta de la violencia de la escuela. Los alumnos socialmente privilegiados en su adscripción a la cultura juvenil mantienen desafíos con la escuela pero dentro de ciertos límites preestablecidos, mientras que los alumnos de las clases populares reaccionan a las experiencias de fracaso por la vía de la afirmación personal y con rebeldía abierta contra la escuela. Para la investigadora Happ Botler (2016: 728), quien realizó un estudio de caso en una escuela pública estadual de la ciudad de Recife, “... muchas veces los adolescentes buscan una forma de autoafirmación, que puede darse por la ruptura de las normas establecidas en la institución escolar. Así, la noción de indisciplina también se relaciona con la de injusticia sufrida/vivida en el ambiente escolar ...”. A similar afirmación arriban Schilling y Angelucci (2016), quienes llevaron a cabo una investigación con estudiantes de secundaria, educación de jóvenes y adultos, y educación superior y profesores de la red pública estadual de educación de São Paulo.

\section{La violencia como modo de relacionarse con el otro}

Como podemos observar, gran parte de las investigaciones de corte cualitativo reveladas centran sus análisis en los sentidos en torno a la violencia en las escuelas que construyen los actores, en nuestro caso particular los estudiantes, como modos de expresión de los sujetos y formas de relacionarse entre los individuos. Es decir, que las situaciones de violencia no son el resultado necesario de una cuestión individual de ciertos sujetos, ni producto de conductas irracionales, así como tampoco una anomalía social. Lo anterior no significa que la violencia en las escuelas no sea tematizada como un problema sobre el cual trabajar, sino que los estudios parten de la premisa de analizar y comprender los sentidos sociales y subjetivos que están en juego en dichas situaciones para poder abordarlas desde distintas aristas.

Uno de los estudios que sintetiza esta línea interpretativa fue realizado tempranamente por los investigadores chilenos García y Madriaza (2005), quienes indagaron sobre los sentidos que los jóvenes 
escolarizados le otorgaban a la violencia. Llevaron a cabo entrevistas individuales y grupos focales a estudiantes de enseñanza media señalados como "agresivos" o "violentos" por las instituciones educativas a las que pertenecían o sus compañeros y, que a su vez, hubieran participado de algún episodio. La hipótesis que guía su trabajo sostiene que la violencia surge de una crisis de sentido existencial cuyo signo sería la dificultad que encuentran los jóvenes para inscribirse subjetivamente a un ethos.

Los investigadores agruparon las respuestas de los estudiantes en once categorías 5 según los sentidos y explicaciones que ellos le encontraban a la violencia. A partir de estas respuestas elaboraron un modelo de análisis en el cual exhiben tres momentos de la violencia: a) como herramienta de conocimiento, b) como búsqueda de reconocimiento y, c) como establecimiento de jerarquías.

Para el primer caso, la alteridad de un otro igual se presenta en algún grado como amenazante en el sentido de que nunca pueden ser predichos los comportamientos de ese otro y la imprevisibilidad se manifiesta como una posibilidad latente. Pero “... esta violencia como herramienta que busca hacer desaparecer cierta amenaza de lo desconocido, apunta no solo reducir lo inaprensible del par, sino también a reducir el desconocimiento, que el propio actor de la violencia tiene de sî" (García y Madriaza, 2005: 174). Los investigadores ponen como ejemplo la situación de los alumnos nuevos que ingresan a un curso.

La violencia como búsqueda de reconocimiento “... se refiere a que frecuentemente estas expresiones cobran un valor, no solo para los implicados directos ... sino también para un tercero que observa y que supuestamente — a ojos del sujeto que agrede-, le da un valor positivo a que surja esta manifestación”(García y Madriaza, 2005: 174).

El tercer momento hace referencia a la posición o rol que ocupan los distintos actores de la violencia en el marco de un grupo. El criterio de jerarquización y eje de conflictividad radica en la superioridad o inferioridad de los estudiantes que interactúan en el espacio escolar.

\footnotetext{
${ }^{5}$ a) Establecer jerarquías. b) Violencia como defensa. c) Resolver un conflicto. d) Pelear por la amistad. e) Catarsis de la violencia. f) Por hacer daño. g) Para entretenerse. h) Por reivindicaciones sociales. i) Para revelarse contra la autoridad. j) Para adquirir bienes. k) No tiene ningún sentido.
} 
Los investigadores concluyen que la violencia no carece de sentido, sino que se anuda a una serie heterogénea de interacciones que construyen un fuerte lazo social de una subcultura emergente con marcos normativos propios que operan a través de la corporalidad, el movimiento, la postura, la voz y la mirada. "Así, esta violencia no es una forma de descontrol, sin límites, caótica por ende; sino que se rige sobre códigos distintos, creados en el debatir mismo del violentarse" (García y Madriaza, 2005: 78).

En esta misma línea, Viscardi (2002) realizó una investigación en Uruguay con el propósito de abordar las prácticas y representaciones del fenómeno de la violencia de los alumnos, profesores, funcionarios y directores en dos liceos públicos de la ciudad de Montevideo. ${ }^{6}$ Desde el punto de vista de la propia institución y sus representantes, la violencia se presenta, por un lado, como un impedimento para realizar las tareas pedagógicas. Por otro lado, desde el punto de vista de los estudiantes, se puede “... poner a la luz las relaciones sociales que se dan en el recinto escolar y su vivencia en tanto relaciones conflictivas que dan en la estructuración de un vínculo violento como modalidad de relación social aceptada, legítima forma de expresión entre pares y con los adultos" (Viscardi, 2002: 32).

La investigadora describe una forma de relacionarse entre los estudiantes que denomina "sociabilidad violenta". Se trata de la presencia de un mecanismo de socialización que pasa por el uso de la fuerza en una lógica ritualizada y que establece jerarquías dentro de los liceos. La relación con la violencia y las estrategias para preservarse de ella forman parte de la vida escolar, generando en los alumnos una pauta de interacción paralela a la del liceo.

Viscardi (2002) sostiene que las situaciones de violencia en las escuelas son la expresión de una crisis de convivencia producto de la dificultad que presenta la institución educativa para socializar a los jóvenes en el marco de los valores que tienen por objetivo impartir, es decir, en términos de los planteos de los investigadores chilenos, se

\footnotetext{
${ }^{6}$ Las prácticas de los estudiantes se analizaron a partir de observaciones participantes dentro y fuera de las aulas, así como también mediante la lectura de sus cuadernos de sanciones disciplinarias. Las representaciones se abordaron mediante entrevistas semiestruturadas grupales para el caso de los estudiantes e individuales en el caso de las autoridades, funcionarios y docentes.
} 
trata de la dificultad que atraviesa la institución para inscribir subjetivamente a los estudiantes en su ethos.

Centrándonos en el tercer momento de la violencia que desarrollan García y Madriaza (2005), podríamos decir que las situaciones conflictivas entre los estudiantes se enmarcan en una forma de sociabilidad de jerarquás donde se busca ser reconocido y también se intenta desprestigiar al otro. Teniendo en cuenta la premisa de que la violencia es un término con fuertes connotaciones morales negativas, en diferentes investigaciones se registró un discurso doble — no doble discurso- entre las percepciones que tenían los estudiantes sobre los comportamiento de los compańeros y los propios, particularmente entre quienes eran tipificados como "violentos".

En Brasil, Abramovay (2005) considera importante indagar las percepciones que tienen los alumnos respecto de los comportamientos de sus compañeros para comprender cómo se construyen las identidades escolares. En el estudio constató que los alumnos tienen una percepción negativa sobre sus compañeros, sobre todo de aquellos que pelean o faltan al respeto. Sin embargo, cuando hablan de los propios comportamientos su visión cambia siendo más positiva.

Las investigadoras interpretan estas percepciones desde dos aristas. Por un lado, sostienen que la visión negativa de sus compañeros es parte de una autorrepresentación incorporada por los jóvenes que se corresponde con una imagen negativa que circula sobre ellos en la sociedad brasileña (Abramovay, 2005). En la misma línea, Kaplan (2011) afirma que en Argentina circula un discurso de sentido común, particularmente difundido por los medios de comunicación, que crean y recrean una condición estigmatizante sobre los jóvenes. Según la autora, la eficacia simbólica de esos discursos se ve reflejada en los testimonios de los mismos estudiantes, en este caso argentinos, que se atribuyen a sí mismo dicha negatividad. Por otro lado, las investigadoras brasileñas afirman que la visión positiva sobre las propias conductas de los alumnos puede deberse a que cuando ellos mismos insultan o cargan a un compañero lo banalizan como algo sin importancia, a pesar de que cuando son destinatarios de esas bromas lo sienten como una ofensa. 
Además de tener en cuenta la autopercepción de los propios entrevistados respecto de actos y actitudes que pueden tener una connotación moral negativa, lo que observamos son dos lógicas discursivas ancladas en el par jerarquizante inferioridad/superioridad, en especial cuando se dialoga con estudiantes que son tipificados como "violentos" por sus compañeros. La primera, de corte proactivo, refiere a la imposición mediante el uso de la fuerza física. La segunda, de corte defensivo, alude a quién utiliza la violencia para resistir o evitar el sometimiento. García y Madriaza (2005) mencionan que al hablar sobre sus pares, los entrevistados señalan la violencia como un instrumento que les sirve a aquéllos para superar a los otros y convertirse en líderes. Pero respecto de ellos mismos, consideran la violencia como un medio para evitar ser tomados de punto y no ocupar una posición inferior dentro del grupo. Los estudiantes argentinos suelen adjudicar el uso de la violencia a aquellos compañeros que buscan "hacerse los malos" (di Napoli, 2013; Tomasini et al., 2013) o "colgarse el cartel de violento" (Kaplan, 2011) para obtener respeto (Paulín, 2015). En la pesquisa desarrollada por Araújo (2001) en una escuela brasilera donde asistían estudiantes que vivían en un vecindario socialmente vulnerable, se observan estos sentidos contrapuestos en torno a la violencia. Mientras algunos estudiantes sostienen que los alumnos que viven en la favela son agresivos y buscan cualquier excusa para pelear, ellos señalan que sus compańeros los tratan mal, los miran y estigmatizan, y es por eso que reaccionan agresivamente.

Kaplan (2011), en sus investigaciones realizadas con estudiantes de escuelas secundarias de la ciudad de La Plata, Provincia de Buenos Aires, aborda la cuestión de la estigmatización de los jóvenes a partir de la atribución de la cualidad de "violentos". La investigadora trabajó con recursos proyectivos que incluyeron fotografías de jóvenes en distintos escenarios y frases emblemáticas. Con el objetivo de indagar sobre las representaciones que tenían sobre sus pares se les preguntó qué imágenes u opiniones tenían sobre los jóvenes que aparecían en las fotografías. Para los estudiantes entrevistados, el corte de pelo, la forma de vestir, las poses y la forma de hablar, podrían funcionar como marcas para identificar al "joven violento". 
La investigadora destaca que el cuerpo es una dimensión fundamental al momento de analizar las relaciones entre los jóvenes y los conflictos que emergen entre sí. Éste es portador de signos que “... fácilmente pueden convertirse en índices dispuestos para orientar la mirada del otro o para ser clasificado sin que uno lo quiera, es decir, operando desde una matriz inconsciente, en términos sociológicos, bajo determinada marca moral o social" (Kaplan, 2013: 49).

Kaplan (2013) destaca que muchos de quienes efectúan los juicios de clasificación portan los mismos rasgos de aquellos que tipifican como violentos; sin embargo, es el "otro" el sujeto violento y amenazante. Esta imagen de "violento", según los estudiantes, formaría parte de un cartel que algunos compańeros intentan colgarse con el objetivo de adquirir prestigio o respeto entre sus pares. De este modo se evidencia que

las categorías de etiquetamiento no pueden ser consideradas como intrínsecas de ciertos sujetos o grupos — ni siquiera la atribución de rasgos físicos- sino que son cualidades percibidas socialmente donde se establece el juego entre grupos superiores e inferiores, entre el sentimiento de mayor y menor valía social, entre auto-imágenes diferenciadas (Kaplan, 2013: 52-53).

Por su parte, Paulín (2013) sostiene que las relaciones conflictivas de los alumnos se configuran en torno a la lucha por el reconocimiento de sus identidades sociales. Los estudiantes de ambas escuelas “... emplean categorías discriminadoras en sus 'jodas' y burlas que operan seleccionando y clasificando a los demás, desde la combinación de tres ejes de comparación: lo racial, la posición social y el género" (Paulín, 2013: 296). A través de la violencia intentan someter o detener al otro reafirmando la identidad personal o grupal.

Los sentidos por la respetabilidad que los jóvenes ponen en juego, así como la lucha por ese respeto, colocan a la violencia como una práctica válida tanto para su construcción como para su defensa. Paulín (2015: 1125) concluye que “... procurarse el respeto a sí mismo sobre el miedo de los demás construye formas frágiles de reconocimiento que deberán sostenerse en forma permanente en ciclos recursivos de violencia”. 


\section{Palabras de cierre}

A lo largo de este artículo presentamos investigaciones cualitativas que, desde la voz de los jóvenes estudiantes, analizan las percepciones en torno a la violencia en las escuelas, los significados que le otorgan, sus causas y manifestaciones, así como también el lugar que le asignan en la construcción subjetiva y en la relación con sus pares.

Resulta necesario recordar que la vida escolar constituye un campo de interconexiones donde se entrelaza lo individual, lo institucional y lo social, y se articulan diversas grupalidades. En este sentido, debe problematizarse y profundizar el análisis de la relación entre las situaciones de violencia y el contexto social en el cual están ubicadas las escuelas, a los fines de no asociar mecánicamente el origen socioeconómico de los estudiantes con determinadas formas de violencia.

Como podemos observar, los "estudiantes" habitan la escuela secundaria no solo en carácter de "alumnos" en cuya formación incorporan saberes, valores y normas sociales, sino también como "jóvenes" que buscan un sentido social a sus vidas. Reunidos en una misma institución conviven varios días a la semana con otros jóvenes de la misma edad forjando vínculos que oscilan entre un polo en el que se construye la alteridad como un "otro hermenéutico", donde prima el encuentro y el afecto, y otro polo donde emerge el conflicto y el miedo a un otro considerado, una "alteridad amenazante". Como vimos a través de diferentes investigaciones, la violencia puede estar presente en ambos polos como un modo de sociabilidad, de estar con el otro.

En muchos casos existe un margen estrecho, ambivalente y difícil de establecer entre dinámicas lúdicas y situaciones conflictivas que devienen en violencia. Existen determinados códigos culturales intrageneracionales, que incluso trasciende las fronteras geográficas, cuya ruptura transforma una situación lúdica en una situación violenta — por ejemplo, el insulto a las madres o los modos de "mirar mal" —. En este marco, consideramos que los vínculos sociales son contingentes y dinámicos y se modifican con el tiempo, en el espacio y de acuerdo con quienes componen las escenas. Por ello, resulta sumamente importante indagar sobre los sentidos que los estudiantes construyen en torno a la violencia en configuraciones particulares no solo para poder comprender 
el fenómeno, sino también para pensar estrategias de intervención situadas y contextualizadas.

El conflicto entre estudiantes puede ser pensado en términos de competencia de grupos de jóvenes por poseer signos distintivos que les permitan conocerse subjetivamente y construir una identidad personal, obtener reconocimiento social y posicionarse jerárquicamente dentro del espacio escolar. Como pudimos observar, el antagonismo, mediante la negación o inferiorización del otro, es una de las formas de autoafirmación subjetiva que muchas veces encuentran los jóvenes. En este marco, las prácticas violentas operan como modo de dominación, como forma de expresarse y/o como atributo identitarios/estigmatizantes.

Finalmente, destacamos que la cuestión emotiva entorno a la violencia constituye una dimensión poco explorada hasta el momento, la cual puede ser una vía de entrada para comprender las formas de interacción, los umbrales de tolerancia y las tensiones que se producen entre los jóvenes en el ámbito escolar. Resulta relevante indagar las diferentes sensibilidades que los estudiantes tienen sobre la violencia a partir de los sentimientos que experimentan en sus vivencias subjetivas.

Los resultados obtenidos en cada una de las investigaciones reseńadas, no solo son de utilidad para nutrir nuevas indagaciones e hipótesis o para visibilizar las particularidades contextuales del fenómeno; sino también constituyen un insumo para pensar dispositivos y programas para abordar la violencia en las escuelas desde una perspectiva socioeducativa no punitiva ni criminalizante.

\section{Bibliografia citada}

Abramovay, M., 2005, "Victimización en las escuelas. Ambiente escolar, robos y agresiones físicas", Revista Mexicana de Investigación Educativa, vol. 10, núm. 26, pp. 833-864.

Abramovay, M., A. L. Cunha y P. P. Calaf, 2009, Revelando tramas, descobrindo segredos: violência e convivência nas escolas, RITLA: Secretaria de Estado da Educação, GDF-Governo do Distrito Federal, Brasília. 
Araújo, C., 2001, "As marcas da violência na constituição da identidade de jovens da periferia", Educação e Pesquisa, vol. 27, núm. 1, pp. 141-160.

Bases de operación del programa nacional escuela segura, 2008, disponible en http://basica.sep.gob.mx/escuelasegura/

Bleichmar, S., 2008, Violencia social-violencia escolar: de la puesta de limites a la construcción de legalidades, Noveduc, Buenos Aires.

Bourdieu, P., 1997, Sobre la televisión, Anagrama, Barcelona.

Brener, G., 2009, "Violencia y escuela como espectáculo. La relación medios-sociedad”, en C. V. Kaplan, Violencia escolar bajo sospecha, Miño y Dávila, Buenos Aires, pp. 199-140.

Charlot, B., 2002, "A violência na escola: como os sociólogos franceses abordam essa questão" Sociologias, vol. 4, núm. 8, pp. 432-443.

Chuquilin Cubas, J. y M. Zagaceta Sarmiento, 2017, "La violencia en las escuelas desde la perspectiva de sus actores. El caso de una escuela secundaria de la Ciudad de México", Revista Educación, vol. 41, núm. 2, pp. 1-19, disponible en https://doi. org/10.15517/revedu.v41i2.21751

Coarite, E. y G. Carrillo, 2014, “Consideraciones sobre la violencia escolar y los conflictos de estudiantes", Revista Integra Educativa, vol. 7, núm. 2, pp. 175-186.

Conde Flores, S. L., 2014, "La violencia y la cultura de la calle entran a la escuela: acciones y reacciones", Sinéctica, núm. 42, pp. 1-21.

D’Angelo, L. A. y D. R. Fernández, 2011, Clima, conflictos y violencia en la escuela, Unicef, Flacso, Buenos Aires.

Di Leo, P., 2009, "Violencias en escuelas medias: discursos y experiencias de jóvenes en la ciudad de buenos aires", Revista Observatorio de juventud, vol. 6, núm. 23, pp. 59-70.

di Napoli, P., 2013, "Violencia, racismo y escuela. El caso de los alumnos tipificados como violentos", Propuesta Educativa, núm. 39, pp. 43-50.

di Napoli, P., 2016a, "La violencia en las escuelas secundarias desde tres perspectivas de análisis. Hacia un estado del arte", Zona Próxima, núm. 24, pp. 61-84, disponible en https://doi. org/10.14482/zp.22.5832 
di Napoli, P., 2016b, Violencias y conflictos en la trama de la vida escolar. Un estudio socioeducativo desde la perspectiva de los jóvenes de educación secundaria, Tesis de doctorado, Universidad de Buenos Aires, Ciudad Autónoma de Buenos Aires.

Duschatzky, S., 2013, "Veo veo...? Qué ves? Percepciones más allá (o más acá) de la violencia", CS Ciencias Sociales, núm. 11, pp. 345.

Duschatzky, S. y C. Corea, 2002, Chicos en banda. Los caminos de la subjetividad en el declive de las instituciones, Paidós, Buenos Aires.

Furlán, A., 2005, "Problemas de indisciplina y violencia en la escuela", Revista Mexicana de Investigación Educativa, vol. 10, núm. 26, pp. 631-639.

García, M. y P. Madriaza, 2005, "Sentido y Sinsentido de la Violencia Escolar: Análisis Cualitativo del Discurso de Estudiantes Chilenos", Psykhe, vol. 14, núm. 1, pp. 165-180.

García-Villanueva, J., A. De la Rosa-Acosta y J. S. Castillo-Valdés, 2012, "Violencia: análisis de su conceptualización en jóvenes estudiantes de bachillerato", Revista Latinoamericana de Ciencias Sociales, Niñez y Juventud, vol. 10, núm. 1, pp. 495-512.

Giordani, J. P., F. Seffner y D. D. Dell'Aglio, 2017, "Violência escolar: percepçóes de alunos e professores de uma escola pública", Psicologia Escolar e Educacional, vol. 21, núm. 1, pp. 103-111, disponible en https://doi.org/10.1590/21753539201702111092

Happ Botler, A. M., 2016, "Injustiça, conflito e violência: um estudo de caso em escola pública de Recife", Cadernos de Pesqui$s a$, vol. 46, núm. 161, pp. 716-732, disponible en https://doi. org/10.1590/198053143676

Kaplan, C. V., 2006, Violencias en plural. Sociología de las violencias en la escuela, Miño y Dávila, Buenos Aires.

Kaplan, C. V., 2009, "Introducción. Las violencias en la escuela desde adentro", en C. V. Kaplan, Violencia escolar bajo sospecha, Miño y Dávila, Buenos Aires, pp. 13-28.

Kaplan, C. V., 2011, "Jóvenes en turbulencia. Miradas críticas contra la criminalización de los estudiantes", Propuesta Educativa, núm. 35, pp. 95-103. 
Kaplan, C. V., 2013, "El miedo a morir joven. Meditaciones de los estudiantes sobre la condición humana”, en C. V. Kaplan, Culturas estudiantiles: sociología de los vínculos en la escuela, Miño y Dávila, Buenos Aires, pp. 45-67.

Kaplan, C. V. y S. García, 2006, "Las violencias en la escuela, en el mundo. Un mapa de los estudios socioeducativos", en C. V. Kaplan, Violencias en plural. Sociología de las violencias en la escuela, Miño y Dávila, Buenos Aires, pp. 105-204.

Kornblit, A. L. (editor), 2008, Violencia escolar y climas sociales, Biblos, Buenos Aires.

Kornblit, A. L., A. M. Mendes Diz y D. Frankel, 1991, "Manifestaciones de violencia en la escuela media”, en F. Lolas Stepke, Agresividad y violencia, Losada, Buenos Aires, pp. 81-101.

Maldonado, M., 2005, "Noviazgo, emotividad y conflicto. Relaciones sociales entre alumnos de la escuela media Argentina", Revista Mexicana de Investigación Educativa, vol. 10, núm. 26, pp. 719-737.

Martín Criado, E., 2005, "La construcción de los problemas juveniles”, Nómadas, núm. 23, pp. 86-93.

Mejía Hernández, J. M. G., 2012, "Incivilidad y violencia: significados de las relaciones sociales entre estudiantes de educación secundaria”, El Cotidiano, núm. 176, pp. 57-65.

Mejía Hernández, J. M. G., 2013, Relaciones sociales y violencias entre adolescentes de secundaria, Tesis de doctorado, Cinvestav, México, disponible en http://www.die.cinvestav.mx/LinkClick.aspx ?fileticket=bYIDezkceQ8\%3D\&tabid=231

Míguez, D. (comp.), 2008, Violencias y conflictos en las escuelas: aproximaciones a una problemática actual, Paidós, Buenos Aires.

Míguez, Daniel, P. Gallo y M. Tomasini, 2015, Las dinámicas de la conflictividad escolar. Procesos y casos en la Argentina reciente, Miño y Dávila, Buenos Aires.

Noel, G., 2009, La conflictividad cotidiana en el escenario escolar: una perspectiva etnográfica, UNSAM Edita, San Martín, Provincia de Buenos Aires. 
Observatorio Argentino de Violencia en las Escuelas, 2008, Violencia en las escuelas: un relevamiento desde la mirada de los alumnos, Ministerio de Educación de la Nación, Buenos Aires.

Paulín, H. L., 2013, Conflictos en la sociabilidad entre jóvenes. Un estudio psicosocial sobre las perspectivas de estudiantes y educadores de escuelas secundarias, Tesis de doctorado no publicada, Universidad Nacional de Córdoba, Córdoba, Argentina.

Paulín, H. L., 2015, “'GANARSE EL RESPETO’: Un análisis de los conflictos de la sociabilidad juvenil en la escuela secundaria”, Revista Mexicana de Investigación Educativa, vol. 20, núm. 67, pp. 1105-1130.

Paulín, H. L. y M. Tomasini, 2008, Conflictos en la escuela secundaria : diversidad de voces y miradas, Universidad Nacional de Córdoba, Córdoba, Argentina.

Paulín, H. L. y M. Tomasini, 2014, Jóvenes y escuela: relatos sobre una relación compleja, Brujas, Córdoba, Argentina, disponible en http://alltitles.ebrary.com/Doc?id=10889574

Potocnjak, M., C. Berger y T. Tomicic, 2011, "Una aproximación relacional a la violencia escolar entre pares en adolescentes chilenos: perspectiva adolescente de los factores intervinientes", Psykhe (Santiago), vol. 20, núm. 2, pp. 39-52, disponible en https:// doi.org/10.4067/S0718-22282011000200004

Ribolla, M. B. y G. A. Fiamenghi Jr., 2007, “Adolescentes na escola: representalccốes sociais sobre violência", Psicologia Escolar e Educacional, vol. 11, núm. 1, pp. 111-121.

Romo, C. C., A. G. Nashiki, A. O. Cervantes y Ú. Z. Rivera, 2016, "La Política Nacional de Convivencia Escolar de México y su impacto en la vida en las escuelas de educación básica”, Posgrado y Sociedad, vol. 14, núm. 1, pp. 2-13.

Saez, V., 2015, "Una mirada a la investigación sobre medios, violencia y escuela”, ENTRAMADO, vol. 11, núm. 1, pp. 136-155, disponible en https://doi.org/10.18041/entramado.2015v11n1.21117 Salles, L. M. F., J. M. A. D. P. e Silva, J. C. R. Castro y C. Fernandez Villanueva, 2014, "Um estudo sobre jovens e violência no espaço escolar”, Psicologia đamp; Sociedade, vol. 26, núm. 1, 
pp. 148-157, disponible en https://doi.org/10.1590/S010271822014000100016

Saucedo, C. L., 2006, "Estudiantes de secundaria: sus apropiaciones de recursos culturales para recrear su condición como jóvenes en la escuela”, Investigación, vol. 11, núm. 29, pp. 403-429.

Schilling, F. y C. B. Angelucci, 2016, "Conflitos, violências, injustiças na escola? Caminhos possíveis para uma escola justa", Cadernos de Pesquisa, vol. 46, núm. 161, pp. 694-715, disponible en https://doi.org/10.1590/198053143675

Spósito, M. P., 2001, "Um breve balanço da pesquisa sobre violência escolar no Brasil", Educação e pesquisa, vol. 27, núm. 1, pp. 87-103.

Tomasini, M., L. Domínguez, y H. Peralta, 2013, Las violencias entre estudiantes desde el punto de vista de sus actores: Una narrativa de la sociabilidad juvenil, Aposta: Revista de ciencias sociales, núm. 58, pp. 2-43.

Torres-Castro, C. B., 2011, "Pandillismo y violencia escolar femenina en el barrio y su proyección a la escuela", Magis. Revista Internacional de Investigación en Educación, vol. 4, núm. 8, pp. 383-398.

Velázquez Reyes, L. M., 2005, "Experiencias estudiantiles con la violencia en la escuela", Revista Mexicana de Investigación Educati$v a$, vol. 10, núm. 26, pp. 739-764.

Viscardi, N., 2002 "Violencia en el espacio escolar en Uruguay: prácticas, respuestas y representaciones", Delito y Sociedad, vol. 1, núm. 17, pp. 31-54, disponible en https://doi.org/10.14409/ dys.v1i17.5427

Viscardi, N. y N. Alonso, 2013, Gramática(s) de la convivencia. Un examen a la cotidianidad escolar y la cultura política en la Educación Primaria y Media en Uruguay, Administración Pública Nacional, Montevideo, Uruguay. 